\section{SAT0582 BONE MARROW LESION TYPE AND PAIN IN KNEE OSTEOARTHRITIS}

T.A. Perry ${ }^{1}$, T.W. O'Neill ${ }^{1,2}$, M.J. Parkes ${ }^{1}$, D.T. Felson ${ }^{1,3}$, R. Hodgson ${ }^{4}$, N. K. Arden ${ }^{5}{ }^{1}$ Arthritis Research UK Centre for Epidemiology, Division of Musculoskeletal and Dermatological Sciences, The University of Manchester, Manchester, ${ }^{2}$ Department of Rheumatology, Salford Royal NHS Foundation Trust, Salford, UK; ${ }^{3}$ Boston University, Boston, USA; ${ }^{4}$ Centre for Imaging Sciences, Institute of Population Health, The University of Manchester, Manchester, ${ }^{5}$ The University of Oxford, Oxford, UK

Background: Bone marrow lesions (BMLs) have been associated with pain in observational studies of knee OA. The aim of this study was to determine whether type of BML (subchondral, with and without regions of well-defined high post-contrast signal, and ligament-based) were associated with knee symptoms.

Objectives: To assess the association between bone marrow lesion volume, including sub-types, and pain in symptomatic knee OA

Methods: Data were collected on a sub-sample of participants who were part of the UK VIDEO study ${ }^{1}$; a 3 year multicentre randomised placebo-controlled trial of vitamin $D$ therapy in patients with symptomatic knee OA. Those recruited to one of the participating centres (Southampton) had contrast enhanced MR imaging (CEMRI) of the index knee performed at one or more annual visits. BML volume was assessed by segmentation of sagittal T1-weighted fat-suppressed post-contrast scans. BMLs were categorised by type (subchondral/ligament-based) and by the presence/absence of regions of well-defined high post-contrast signal (for the subchondral BMLs only). As part of the trial, subjects completed the WOMAC questionnaire which included questions on pain, function and stiffness. We used random-effects multiple linear panel regression, adjusting for variance between follow-up visits to explore the relationship between BML volume and the WOMAC pain and function subscales. This approach is preferred over cross-sectional analyses, as it makes use of all available observations from study visits, and controls for within-patient correlations appropriately.

Results: In this analysis, we included 50 subjects who had had a baseline MRI and at least one other MRI performed. The mean age of the subjects was 63.3 $(S D \pm 6.5)$ years and $74 \%$ were female. After adjusting for variation between visits, there was no significant association between total BML volume and WOMAC pain when the BMLs were stratified by type; total subchondral $B M L$ volume $(b=41.5$ $\mathrm{mm}^{3} ; 95 \% \mathrm{Cl}-19.35$ to 102.37$)$ or total ligament-based $\mathrm{BML}$ volume $(b=9.1$ $\mathrm{mm}^{3} ; 95 \% \mathrm{Cl}-6.39$ to 24.59 ). Further, we did not observe an association between pain and volume of subchondral BMLs with or without regions of welldefined high post-contrast signal; (i) total mixed subchondral oedema-like BMLs containing regions of well-defined high signal $\left(b=39.45 \mathrm{~mm}^{3} ; 95 \% \mathrm{Cl}-3.93\right.$ to $82.83)$ or (ii) subchondral oedema-like BML volume $\left(b=-4.16 \mathrm{~mm}^{3} ; 95 \% \mathrm{Cl}\right.$ -53.49 to 45.18 ), after adjusting for variation between visits. There was, however, a significant association between the volume of subchondral regions of welldefined high signal and both WOMAC pain $\left(b=2.19 \mathrm{~mm}^{3} ; 95 \% \mathrm{Cl} 0.88\right.$ to 3.49$)$ and function ( $b=1.61 \mathrm{~mm}^{3} ; 0.37$ to 2.84 ).

Conclusions: In this analysis, an increased volume of regions of well-defined high signal intensity on post-contrast scans within subchondral BMLs was associated with knee pain and function.

\section{REFERENCE:}

[1] Arden NK, Cro S, Sheard S, Dore CJ, Bara A, Tebbs SA, et al. The effect of vitamin D supplementation on knee osteoarthritis, the VIDEO study: a randomised controlled trial. Osteoarthritis Cartilage 2016 Nov;24(11):185866 .

Disclosure of Interest: None declared

DOI: 10.1136/annrheumdis-2018-eular.2875

\section{SAT0583 \\ THE IMMEDIATE EFFECT OF A SOFT KNEE BRACE ON DYNAMIC KNEE INSTABILITY IN PERSONS WITH KNEE OSTEOARTHRITIS}

T. Cudejko ${ }^{1}$, M. van der Esch ${ }^{2}$, J. Schrijvers ${ }^{1}$, R. Richards ${ }^{1}$, T. Wrigley ${ }^{3}$, J. van den Noort ${ }^{1}$, M. van der Leeden ${ }^{2}$, L.D. Roorda ${ }^{2}$, W. Lems ${ }^{1}$, J. Harlaar ${ }^{1}$, J. Dekker ${ }^{1} .{ }^{1}$ VU University Medical Center, ${ }^{2}$ Amsterdam Rehabilitation Research Center, Reade, Amsterdam, Netherlands; ${ }^{3}$ University of Melbourne, Melbourne, Australia

Background: Wearing a soft knee brace has been shown to reduce self-reported knee instability in persons with knee osteoarthritis $(\mathrm{OA}) .{ }^{1}$ There is a need to assess whether a soft knee brace has a beneficial effect on objectively assessed dynamic knee instability as well.

Objectives: The aims of the study were: (i) to evaluate the immediate effect of a soft knee brace on dynamic knee instability, and (ii) to assess the difference in effect between a tight and a non-tight knee brace in persons with knee $O A$.

Methods: A within-subject cross-over design was used, comparing wearing a soft knee brace with not wearing a soft knee brace, and comparing wearing a tight brace (standard fit) with wearing a non-tight brace (one size larger). The order of brace type was randomised. Participants walked, both without and with the brace, on a treadmill, which is integrated in the GRAIL system, placed in a virtual reality environment (GRAIL system, MOTEKForce Link, The Netherlands). Participants were subjected to two tasks: (i) level walking and (ii) walking with mechanical perturbations on the treadmill. Mechanical perturbations on the treadmill comprised five lateral and five medial translations ( $2 \mathrm{~cm}$ displacements) of the treadmill belts occurring during $20 \%-50 \%$ of the gait cycle. During the walking trials, 3D movement of the lower legs, pelvis and trunk were captured via markers on anatomical landmarks at $100 \mathrm{~Hz}$ using a motion-capture system (Vicon, Oxford, United Kingdom). The outcome measure was dynamic knee instability, expressed by the Perturbation Response (PR), i.e. a biomechanics based measure reflecting deviation in the mean knee varus-valgus angle after a controlled mechanical perturbation, standardised to the mean (standard deviation) varus-valgus angle during leve walking. Lower PR values indicate less deviation in the mean varus/valgus angle. Linear mixed-effect model analysis was used to evaluate the effect of a brace on dynamic knee instability.

Results: Thirty-eight persons with knee OA and self-reported knee instability from the Amsterdam Osteoarthritis Cohort participated in the study. Wearing a brace significantly reduced the PR compared to not wearing a brace $(p<0.05)$. The PR value reduced from 0.48 when not wearing a brace to 0.32 when wearing a brace. This means that wearing a brace resulted in a reduction of $33 \%$ in dynamic knee instability compared to not wearing a brace. There was no difference between a non-tight and a tight brace $(\mathrm{p}>0.05)$.

Conclusions: This study is the first to report that wearing a soft brace results in an improvement of objectively assessed dynamic knee instability, beyond the previously reported subjective improvement.

\section{REFERENCE:}

[1] Cudejko T, van der Esch M, van der Leeden M, van den Noort JC, Roorda LD, Lems W, Twisk J, Steultjens M, Woodburn J, Harlaar J, Dekker J: The immediate effect of a soft knee brace on pain, activity limitations, selfreported knee instability, and self-reported knee confidence in patients with knee osteoarthritis. Arthritis Res Ther 2017;19:260.

Disclosure of Interest: None declared

DOI: 10.1136/annrheumdis-2018-eular.5467

\section{SAT0584 CLINICAL VALIDATION OF TWO PANELS OF BIOMARKERS TO PREDICT SYMPTOMATIC DRUGS RESPONSE IN KNEE OA}

V. Calamia ${ }^{1}$, F. Picchi ${ }^{1}$, I. Rego ${ }^{1}$, M. Camacho ${ }^{1}$, L. González ${ }^{1}$, P. FernándezPuente $^{1}$, M. Herrero ${ }^{2}$, H. Martínez ${ }^{2}$, J. Vergés ${ }^{3}$, C. Ruiz-Romero ${ }^{1}$, F.J. Blanco ${ }^{1}$ ${ }^{1}$ INIBIC, La Coruña; ${ }^{2}$ Bioiberica S.A.U; ${ }^{3}$ OAFI Foundation, Barcelona, Spain

Background: The prediction of drug responses based on the analysis of multiple clinical variables and omics data is mandatory for accomplishing the promise of precision medicine in rheumatology.

Objectives: Integrating clinical-radiological-analytical variables and proteomics data for predicting patient's response to different treatments, in order to optimise therapeutic outcomes in OA.

Methods: A panel of 10 serum proteins potentially useful to predict $O A$ patient's response was qualified using ELISA Kits in the whole Multicentre Osteoarthritis interVEntion trial with Sysadoa (MOVES) cohort. Patients were classified as responders $(\mathrm{R})$ and non-responders (NR), either to Chondroitin sulfate/Glucosamine hydrochloride (CS+GH; Droglican, Bioiberica, Spain) or Celecoxib, after 6 months of treatment. Logistic regression analyses, adjusted by confounder variables previously reported as significant in bivariate approaches, were used to analyse the contribution of the measured proteins to our prediction models of drug response in knee OA. Appropriate receiver-operating-characteristics $(\mathrm{ROC})$ curves were also calculated.

Results: In the discovery phase of the study, two different panels of putative predictive biomarkers useful to stratify $O A$ patients according to their unique protein profiles was identified by shotgun proteomics $(n=80)$. In the verification phase, the panel of 6 proteins specific for Droglican treatment (APOA2, APOA4, APOH, ITIH1, C4BPa and ORM2), and the panel of 4 proteins specific for Celecoxib treatment $(\alpha 2 \mathrm{HS}$, SHBG, CD5L and TSP1) were verified in a larger cohort of OA patients ( $n=262$ for $C S+G H$ group and $n=244$ for Celecoxib group) by ELISA assays. In the qualification phase, the sensitivity and specificity of a panel of 4 validated proteins (ORM2, APOA2, ITIH1, and TSP1) were tested in blind in the whole MOVES cohort at baseline $(n=506)$. In CS+GH group, only ORM2 levels were significantly lower in responders compared to non-responders (R: 192,8 ug/ $\mathrm{mL}$ vs NR: $261,6 \mathrm{ug} / \mathrm{mL} ; \mathrm{p}=0,042$ ), while no statistically significant differences were found in the Celecoxib group. Five clinical and two analytical parameters recorded at baseline significantly influence patients' response regardless of treatment. Notably, if we include ORM2 as covariate, we found a specific interaction 